\title{
A Note on Fixed Point Theorems in Shells of Banach Spaces for Weakly Condensing Operators
}

\author{
D. O'Regan
}

\begin{abstract}
New fixed point results are presented for weakly condensing maps. The analysis relies on a continuation theorem [3], recently presented for such operators.
\end{abstract}

Keywords: Fixed points, weakly condensing, measures of weak non-compactness

AMS subject classification: $47 \mathrm{H} 10$

\section{Introduction}

In [3], motivated by work of De Blasi and Emmanuele, we obtained a topological transversality theorem for weakly condensing operators. Various fixed point results were also established. We continue these studies here and obtain new fixed point results for weakly condensing operators in shells of Banach spaces. By a shell $S$ of a Banach space $E$ we mean a set of the form $S=W \backslash V$, where $V$ and $W$ are non-empty subsets of $E$ with $V \subset W$. Our results were motivated, in part, by well known results for compact operators in cones of Banach spaces (see [6] and its references). We will also show that the ideas used for weakly condensing operators extend to many other classes of maps defined on locally convex Hausdorff linear topological spaces.

For the remainder of this section we gather together some definitions and known facts. Let $\Omega_{E}$ be the system of bounded subsets of a Banach space $E$ and let $K^{w}$ be the family of all weakly compact subsets of $E$. Also, let $B$ be the closed unit ball of $E$. The De Blasi measure of weak non-compactness (see [3]) is the map $w: \Omega_{E} \rightarrow[0, \infty]$ defined by

$$
w(X)=\inf \left\{t>0: \text { there exists } Y \in K^{w} \text { with } X \subseteq Y+t B\right\} \quad\left(X \in \Omega_{E}\right) .
$$

Suppose $F: Z \subseteq E \rightarrow E$ maps bounded sets into bounded sets and is weakly continuous. For such an $F$ we say the following:

(i) $F$ is a $w$-Lipschitzian map with constant $k \geq 0$ if $w(F(X)) \leq k w(X)$ for all bounded sets $X \subseteq Z$.

(ii) $F$ is a $w$-condensing map if $F$ is $w$-Lipschitzian with $k=1$ and $w(F(X))<w(X)$ for all bounded sets $X$ with $w(X) \neq 0$.

D. O'Regan: University College Galway, Department of Mathematics, Galway, Ireland 
Remark. $F$ being $w$-Lipschitzian with $k=1$ can be removed from the definition of $w$-condensing and the results of this paper are again true.

Finally, we state the Eberlein-Śmulian theorem (this will be used in Section 2).

Theorem 1.1. Suppose $K$ is weakly closed in a Banach space $E$. Then the following assertions are equivalent:

(i) $K$ is weakly compact.

(ii) $K$ is weakly sequentially compact.

\section{Fixed point theory}

Let $E$ be a Banach space and let $Q, C$ be closed bounded convex subsets of $E$ with $Q \subseteq C$. Also, let $U$ be a weakly open subset of $Q$. For notational purposes let $\overline{U^{w}}$ denote the weak closure of $U$ in $Q$ and $\partial_{Q} U$ the weak boundary of $U$ in $Q$. Following [3] we let $P_{\partial_{Q} U}\left(\overline{U^{w}}, C\right)$ denote the set of all $w$-condensing mappings (in our terminology this automatically means weakly continuous as well) $F: \overline{U^{w}} \rightarrow C$ such that $F$ is fixed point free on $\partial_{Q} U$. A map $F \in P_{\partial_{Q} U}\left(\overline{U^{w}}, C\right)$ is essential in $P_{\partial_{Q} U}\left(\overline{U^{w}}, C\right)$ if every map in $P_{\partial_{Q} U}\left(\overline{U^{w}}, C\right)$ which agrees with $F$ on $\partial_{Q} U$ has a fixed point. Otherwise $F$ is inessential in $P_{\partial_{Q} U}\left(\overline{U^{w}}, C\right)$, i.e. there exists a fixed point free $G \in P_{\partial_{Q} U}\left(\overline{U^{w}}, C\right)$ with $G=F$ on $\partial_{Q} U$. Two maps $F, G \in P_{\partial_{Q} U}\left(\overline{U^{w}}, C\right)$ are homotopic in $P_{\partial_{Q} U}\left(\overline{U^{w}}, C\right)$ written $F \cong G$ in $P_{\partial_{Q} U}\left(\overline{U^{w}}, C\right)$ if there is a $w$-condensing [3] mapping $N: \overline{U^{w}} \times[0,1] \rightarrow C$ such that $N_{t}(u)=N(u, t): \overline{U^{w}} \rightarrow C$ belongs to $P_{\partial_{Q}} U\left(\overline{U^{w}}, C\right)$ for each $t \in[0,1]$ and $N_{0}=F, N_{1}=G$.

The following two results are found in [3].

Theorem 2.1. Let $E, Q, C$ and $U$ be as above. Suppose $F$ and $G$ are two maps in $P_{\partial_{Q} U}\left(\overline{U^{w}}, C\right)$ such that $F \cong G$ in $P_{\partial_{Q} U}\left(\overline{U^{w}}, C\right)$. Then $F$ is essential in $P_{\partial_{Q} U}\left(\overline{U^{w}}, C\right)$ if and only if $G$ is essential in $P_{\partial_{Q} U}\left(\overline{U^{w}}, C\right)$.

Theorem 2.2. Let $E, Q, C$ and $U$ be as above, with $u_{0} \in U$. Then the constant map $F: \overline{U^{w}} \rightarrow u_{0}$ is essential in $P_{\partial_{Q}} U\left(\overline{U^{w}}, C\right)$.

The next result is immediate from Theorems 2.1 and 2.2 .

Theorem 2.3. Let $E$ be a Banach space and $Q, C$ are closed bounded convex subsets of $E$ with $Q \subseteq C$. Also, $U$ is a weakly open subset of $Q$ with $u_{0} \in U$. In addition assume the following:

$$
\left.\begin{array}{l}
h: \overline{U^{w}} \times[0,1] \rightarrow C \text { is a w-condensing map such that } \\
h(x, 0)=u_{0} \text { for all } x \in \overline{U^{w}} \text { and, for each } t \in[0,1], \\
x \neq h_{t}(x) \text { for } x \in \partial_{Q} U \text { where } h_{t}(\cdot)=h(\cdot, t) .
\end{array}\right\}
$$

Then $h(\cdot, 1)$ has a fixed point in $U$.

Theorem 2.3 gives us a new fixed point result for $w$-Lipschitzian maps with $k=1$. 
Theorem 2.4. Let $E \cdot$ be a Banach space and $Q, C$ are closed bounded convex subsets of $E$ with $Q \subseteq C$. Also, $U$ is a weakly open subset of $Q, 0 \in U$ and $\overline{U^{w}}$ is weakly compact. Suppose $F: \overrightarrow{U^{w}} \rightarrow C$ is a $w$-Lipschitzian map with $k=1$ and $x \neq \lambda F(x)$ for $\lambda \in[0,1]$ and $x \in \partial_{Q} U$. Then $F$ has a fixed point.

Proof. For each $n \in\{2,3, \ldots .$.$\} define$

$$
F_{n}=\left(1-\frac{1}{n}\right) F: \overline{U^{w}} \rightarrow C .
$$

Now $F_{n}$ is a $w$-condensing map. Let

$$
h(x, t)=t F_{n}(x) \quad \text { for }(x, t) \in \overline{U^{w}} \times[0,1] .
$$

It is easy to check that $h$ satisfies (2.1) with $u_{0}=0$. Theorem 2.3 implies that $F_{n}$ has a fixed point $x_{n} \in \overline{U^{w}}$, i.e. $x_{n}=\left(1-\frac{1}{n}\right) F\left(x_{n}\right)$. Now since $F\left(\overline{U^{w}}\right)$ is bounded we have

$$
x_{n}-F\left(x_{n}\right)=-\frac{1}{n} F\left(x_{n}\right) \rightarrow 0 \quad \text { as } n \rightarrow \infty .
$$

So in particular

$$
x_{n}-F\left(x_{n}\right) \rightarrow 0 \quad: \text { as } n \rightarrow \infty
$$

where $\rightarrow$ denotes weak convergence. The Eberlein-S̆mulian theorem guarantees a subsequence $S$ of integers and an element $u \in \bar{U}^{w}$ with $x_{n}-u$ as $n \rightarrow \infty$ in $S$. Now since $F: \overline{U^{w}} \rightarrow C$ is. weakly continuous we have $F\left(x_{n}\right)-F(u)$ as $n \rightarrow \infty$ in $S$ and so

$$
x_{n}-F\left(x_{n}\right) \rightarrow u-F(u) \quad \text { as } n \rightarrow \infty \text { in } S .
$$

This together with (2.2) yields $u=F(u)$

Remark. The result of Theorem 2.4 is again true if $\overline{U^{w}}$ weakly compact is replaced by the condition: if $\left\{x_{n}\right\} \in \overline{U^{w}}$ and $x_{n}-F\left(x_{n}\right) \rightarrow 0$ as $n \rightarrow \infty$, then there exists $x \in \overline{U^{w}}$ with $x=F(x)$.

We now establish fixed point theory in shells of Banach spaces. Our theory relies on the following fixed point result.

Theorem 2.5. Let $E$ be a Banach space and $Q, C$ are closed bounded convex subsets of $E$ with $Q \subseteq C$. Let $U_{1}$ and $U_{2}$ be two weakly open subsets of $Q$. with $\overline{U_{1}^{w}} \subset U_{2}$ (proper) and $F: \overline{U_{2}^{w}} \rightarrow C$ is a $w$-condensing map. In addition suppose the following:

$$
\begin{aligned}
& x \neq F(x) \text { for } x \in \partial_{Q} U_{1} \cup \partial_{Q} U_{2} \\
& F: \overline{U_{1}^{w}} \rightarrow C \text { is inessential in } P_{\partial_{Q}} U_{1}\left(\overline{U_{1}^{w}}, C\right) \text { (i.e. }\left.F\right|_{\overline{U_{1}^{w}}} \text { is inessential) } \\
& F: \overline{U_{2}^{w}} \rightarrow C \text { is essential in } P_{\partial_{Q} U_{2}}\left(\overline{U_{2}^{w}}, C\right) .
\end{aligned}
$$

Then $F$ has at least one fixed point in $U_{2} \backslash \overline{U_{1}^{w}}$.

Proof. Suppose $F$ has no fixed points in $U_{2} \backslash \overline{U_{1}^{w}}$. Now (2.4) implies that there is a $w$-condensing map $\theta: \overline{U_{1}^{w}} \rightarrow C$ with $\theta=F$ on $\partial_{Q} U_{1}$ and $x \neq \theta(x)$ for $x \in \overline{U_{1}^{w}}$. Define the mapping $\Phi: \overline{U_{2}^{w}} \rightarrow C$ by

$$
\Phi(x)= \begin{cases}F(x) & \text { for } x \in \overline{U_{2}^{w}} \backslash \overline{U_{1}^{w}} \\ \theta(x), & \text { for } x \in \overline{U_{1}^{w}}\end{cases}
$$

Now $\Phi$ is a $w$-condensing map and $\Phi$ has no fixed points on $\overline{U_{2}^{w}}$ (since $\theta$ has no fixed points on $\overline{U_{1}^{w}}$ and $F$ has no fixed points on $\left.U_{2} \backslash \overline{U_{1}^{w}}\right)$. This contradicts (2.5) 
Theorem 2.5 now guarantees the following applicable result.

Theorem 2.6. Let $E$ be a Banach space and $Q, C$ are closed bounded convex subsets of $E$ with $Q \subseteq C$. Let $U_{1}$ and $U_{2}$ be two weakly open subsets of $Q$ with $\overline{U_{1}^{w}} \subset U_{2}$ (proper) and $0 \in U_{1}$. In addition assume the following:

$$
\left.\begin{array}{c}
N: \overline{U_{2}^{w}} \times[0,1] \rightarrow C \text { is w-condensing with } N(x, 0)=0 \text { for all } x \in \overline{U_{2}^{w}} \\
\text { and, for each } t \in[0,1], x \neq N_{t}(x) \text { for all } x \in \partial_{Q} U_{2} \text { where } N_{t}(\cdot)=N(\cdot, t)
\end{array}\right\}
$$

Then $N(\cdot, 1)$ has a fixed point in $U_{2} \backslash \overline{U_{1}^{w}}$.

Proof. From Theorem 2.2 we know that the zero map is essential in $P_{\partial_{Q}} U_{2}\left(\overline{U_{2}^{w}}, C\right)$. Then (2.6) together with Theorem 2.1 implies

$$
N(\cdot, 1): \overline{U_{2}^{w}} \rightarrow C \quad \text { is essential in } P_{\partial_{Q} U_{2}}\left(\overline{U_{2}^{w}}, C\right)
$$

Also, (2.9) (and also (2.7)) implies that $H(x, 0)$ is inessential in $P_{\partial_{Q} U_{1}}\left(\overline{U_{1}^{w}}, C\right)$ (this follows immediately from the definition of inessential in $P_{\partial_{Q} U_{1}}\left(\overline{U_{1}^{w}}, C\right)$ ). This together with (2.7), (2.8) and Theorem 2.1 yields

$$
N(\cdot, 1)=H(\cdot, 1): \overline{U_{1}^{w}} \rightarrow C \quad \text { is inessential in } P_{\partial_{Q} U_{1}}\left(\overline{U_{1}^{w}}, C\right) .
$$

Thus the conditions of Theorem 2.5 hold

Our next theorem was motivated by results for compact operators defined on cones of Banach spaces.

Theorem 2.7. Let $E$ be a Banach space and $Q, C$ are closed bounded convex subsets of $E$ with $Q \subseteq C$. Let $U_{1}$ and $U_{2}$ be two weakly open subsets of $Q$ with $\overline{U_{1}^{w}} \subset U_{2}$ (proper) and $0 \in U_{1}$. Suppose $F: \overline{U_{2}^{w}} \rightarrow C$ is a w-condensing map and assume the following:

$$
x \neq \lambda F(x) \text { for } \lambda \in[0,1] \text { and } x \in \partial_{Q} U_{2} \text {. }
$$

There exists a $v \in Q \backslash\{0\}$ with $x \neq F(x)+\delta v$ for $\delta \geq 0$ and $x \in \partial_{Q} U_{1}$.

Then $F$ has a fixed point in $\overline{U_{2}^{w}} \backslash U_{1}$.

Proof. Now $x \neq F(x)$ for $x \in \partial_{Q} U_{2} \cup \partial_{Q} U_{1}$ (see (2.12) with $\lambda=1$ and (2.13) with $\delta=0$ ). Choose $M>0$ such that $\|F(x)\| \leq M$ for all $x \in \overline{U_{1}^{w}}$ and choose $\delta_{0}>0$ such that

Now let

$$
\left\|\delta_{0} v\right\|>M+\sup \left\{\|x\|: x \in \overline{U_{1}^{w}}\right\} \text {. }
$$

$$
N(\cdot, t)=t F(\cdot) \quad \text { and } \quad H(\cdot, t)=F(\cdot)+(1-t) \delta_{0} v .
$$

Clearly, (2.12) and (2.13) imply that (2.6) and (2.7) in Theorem 2.6 are satisfied. Also, $(2.8)$ is trivially true and (2.14) implies that (2.9) is satisfied (note $\left.H(x, 0)=F(x)+\delta_{0} v\right)$. The result now follows from Theorem 2.6 
Remark. The results in this section could be extended to multivalued maps by combining the ideas in this paper with those in $[3,5]$. Let $E$ be a Banach space and $F: Z \subseteq E \rightarrow 2^{E}$ where $2^{E}$ denotes the family of all non-empty subsets of $E$. Suppose $F: Z \rightarrow 2^{E}$ maps bounded sets into bounded sets and is weakly upper semicontinuous [5] (i.e, the set $F^{-1}(A)$ is weakly closed for any weakly closed set $A$ ). For such situation we say the following:

(i) $F$ is a $w$-Lipschitzian map with constant $k \geq 0$ if $w(F(X)) \leq k w(X)$ for all bounded sets $X \subseteq Z$.

(ii) $F$ is a $w$-condensing map if $F$ is $w$-Lipschitzian with $k=1$ and $w(F(X))<w(X)$ for all bounded sets $X$ with $w(X) \neq 0$.

Let $Q$ be a weakly complete convex subset of $E$ and $U$ a weakly open subset of $Q$. Now let $W P_{\partial_{Q} U}\left(\overline{U^{w}}, E\right)$ denote the set of all $w$-condensing mappings (in our terminology this automatically means weakly upper semicontinuous as well) $F: \overline{U^{w}} \rightarrow C K(E)$ such that $F$ is fixed point free on $\partial_{Q} U$ where $C K(E)$ denotes the family of all non-empty weakly compact, convex subsets of $E$. There are also obvious analogue definitions for essential in $W P_{\partial_{Q} U}\left(\overline{U^{w}}, E\right)$ and $\cong$ in $W P_{\partial_{Q} U}\left(\overline{U^{w}}, E\right)$. Essentially the same reasoning as in this section together with the results in [5: Section 3] would establish the analogue of Theorems 2.5 - 2.7 for maps in $W P_{\partial_{Q} U}\left(\overline{U^{w}}, E\right)$.

Finally, we would like to show that the ideas in this paper extend to many other classes of maps. For the remainder of this section assume $E$ is a Fréchet space (a complete metrizable locally convex linear topological space). Let $P$ be a defining system of seminorms on $E, C$ a closed convex subset of $E$, and $U$ an open subset of $C$. Further, $K_{\partial U}(\bar{U}, C)$ denotes the family of all upper semicontinuous, $P$-concentrative $[4,5]$ maps $F: \bar{U} \rightarrow A(C)$ with $F(\bar{U})$ a subset of a bounded set in $C$ and such that $F$ is fixed point free on $\partial U$ were $\bar{U}$ and $\partial U$ denote the closure and boundary of $U$ in $C$, respectively, and $A(C)$ denotes the family of all non-empty compact, $R_{\delta}$ (or, more generally, acyclic [2]) subsets of $C$. There are obvious analogue definitions for essential in $K_{\partial U}(\bar{U}, C)$ and $\cong$ in $K_{\partial U}(\vec{U}, C)$. Essentially the same reasoning as in [5] establishes the following result (see also [2]).

Theorem 2.8. Let $E, C$ and $U$ be as above. Suppose $F$ and $G$ are two maps in $K_{\partial U}(\bar{U}, C)$ such that $F \cong G$ in $K_{\partial U}(\bar{U}, C)$. Then $F$ is essential in $K_{\partial U}(\bar{U}, C)$ if and only if $G$ is essential in $K_{\partial U}(\bar{U}, C)$.

The analogue of Theorem 2.2 is immediate also from the ideas in [5] and a result of Fitzpatrick and Petryshyn [1: Theorem 1].

Theorem 2.9. Let $E, C$ and $U$ be as above with $u_{0} \in U$. Then the constant map $F: \bar{U} \rightarrow\left\{u_{0}\right\}$ is essential in $K_{\partial U}(\bar{U}, C)$.

Essentially the same reasoning as before establishes analogues of Theorems 2.5 2.7. For completeness we state the analogue of Theorem 2.7 in this setting.

Theorem 2.10. Let $E$ be a Fréchet space and $C$ a closed convex subset of $E$. Let $U_{1}$ and $U_{2}$ be two open subsets of $C$ with $\overline{U_{1}} \subset U_{2}$ (proper) and $0 \in U_{1}$. Suppose 
$F: \overline{U_{2}} \rightarrow A(C)$ is a upper semicontinuous $P$-concentrative map with $F\left(\overline{U_{2}}\right)$ a subset of a bounded set in $C$ and assume the following:

$x \notin \lambda F(x)$ for $\lambda \in[0,1]$ and $x \in \partial U_{2}$.

There exists $a v \in C \backslash\{0\}$ with $x \notin F(x)+\delta v$ for $\delta \geq 0$ and $x \in \partial U_{1}$.

Then $F$ has a fixed point in $\overline{U_{2}} \backslash U_{1}$.

\section{References}

[1] Fitzpatrick, P. M. and W. V. Petryshyn: Fixed point theorems for multivalued noncompact acyclic mappings. Pac. J. Math. 54 (1974), 17 - 23.

[2] Gorniewicz, L., Granas, A. and W. Kryszewski: Sur la méthode l'homotopie dans la théorie des points fixes pour les applications multivoques. Partie 1: Transversalité topologique. C.R. Acad. Sci. Paris 307 (1988), 489 - 492.

[3] O'Regan, D.: A continuation method for weakly condensing operators. Z. Anal. Anw. 15 (1996), 565 - 578.

[4] O'Regan, D.: Continuation fixed point theorems for locally convex linear topological spaces. Mathl. Comput. Modelling 24 (1996), 57 - 70.

[5] O'Regan, D.: Fixed points for set valued mappings in locally. convex linear topological spaces. To appear.

[6] Precup, R.: On some fixed point theorems of Deimling. Nonlin. Anal. 23 (1994), 1315 1320.

[7] Tarafdar, E. and R. Vyborny:-Fixed point theorems for condensing multivalued mappings on a locally convex topological space. Bull. Austral. Math. Soc. 12 (1975), $161-170$.

Received 08.11.1996 